\title{
A FINITE ELEMENT CONSTITUTIVE UPDATE SCHEME FOR ANISOTROPIC, VISCOELASTIC SOLIDS EXHIBITING NON-LINEARITY OF THE SCHAPERY TYPE
}

\author{
HARRISON POON ${ }^{\dagger}$ AND M. FOUAD AHMAD ${ }^{*, \ddagger}$
}

National Center for Supercomputing Applications, University of Illinois at Urbana-Champaign, Urbana, IL 61801, U.S.A.

\section{SUMMARY}

This study presents a new scheme for performing integration point constitutive updates for anisotropic, small strain, non-linear viscoelasticity, within the context of implicit, non-linear finite element structural analysis. While the basic scheme has been presented earlier by the authors for linear viscoelasticity, the present work illustrates the generality of the underlying fundamentals by extending to Schapery's non-linear model. The method features a judicious choice of state variables, a stable backward Euler integration step, and a consistent tangent operator. Its greatest strength lies in ready incorporation into existing FEM codes. Numerical examples involving homogeneous stress states such as uniaxial extension and simple shear, and non-uniform stress states such as a beam under tip load, were carried out by incorporating the present scheme into a general purpose FEM package. Excellent agreement with analytical results is observed. Copyright (C) 1999 John Wiley \& Sons, Ltd.

KEY WORDS: non-linear; anisotropy; viscoelasticity; finite elements

\section{INTRODUCTION}

The finite element modelling of viscoelastic behaviour has been widely reported in the literature. The vast majority, however, deals with the isotropic case where there are only two distinct sets of relaxation behaviour - one associated with the shear modulus and the other with bulk modulus. The reader is referred to References 1-5, for samples of this line of work. A more comprehensive literature review may be found in References 6 and 7.

Many important materials in engineering exhibit anisotropic viscoelastic behaviour. An example is thermosetting matrix composites undergoing autoclave curing. Depending on the arrangement of fibres, the level of anisotropy is at least transversely isotropic, with five distinct modulus components, each with its individual relaxation behaviour. Up to 21 components can be expected in the truly anisotropic case.

Yi et al. ${ }^{8-14}$ have carried out finite element simulations with anisotropic viscoelasticity, mostly dealing with curing-induced residual stress calculations of thermoset composites. Their finite element algorithm is detailed in Reference 6. In brief, the approach starts from the constitutive

\footnotetext{
*Correspondence to: M. Fouad Ahmad, EDS, 5401 Gateway Center, Flint, MI 48507, U.S.A.

${ }^{\dagger}$ Currently with Hibbitt, Karlsson \& Sorensen (HKS), Inc.

‡Currently with Electronic Data Systems (EDS) Corporation
}

CCC 0029-5981/99/362027-15\$17.50

Copyright (C) 1999 John Wiley \& Sons, Ltd. 
relation, applies the viscoelastic version of the variational principle, invokes finite element discretization, and arrives at a system of integral equations in the nodal unknowns. By employing techniques such as the Laplace transform, it is possible to march forward in time by keeping the solutions from the previous two time steps, thus obtaining the transient time history without too much memory burden.

Zocher et al. $^{7}$ presented a novel finite element scheme for linear anisotropic viscoelasticity. Rather than dealing with integral equations, the authors introduced a smart integration point constitutive update algorithm whereby the stress increment is separated into a part which is linear in the strain increment, and a non-linear contribution which can be computed from stored results in the previous increment. Consequently, the overall incremental virtual work equation is linear in the displacement increment, bypassing the need for Newton iterations and the consistent tangent operator. However, the assumption of a single reduced time for all directions may be physically unrealistic for certain materials, such as fibre-reinforced composites.

Poon and $\mathrm{Ahmad}^{15}$ have presented an alternate integration point constitutive update algorithm very similar in spirit to that of Zocher et al. ${ }^{7}$ Following Yi et al., ${ }^{8-14}$ the reduced time is directional dependent. An intelligent choice of state variables leads to evolution laws in the form of linear first-order ODEs, the time integration of which can be achieved by a variety of common methods, e.g. backward Euler. Most importantly, the method is fully compatible with standard non-linear finite element analysis, where the incremental virtual work equation is non-linear in the displacement increment, requiring techniques such as Newton-Raphson iterations and consistent tangent operators.

The original algorithm of Poon and $\mathrm{Ahmad}^{15}$ addresses linear viscoelasticity. The key algebraic structure and the basic steps in the algorithmic development are general enough to accommodate non-linear viscoelastic behaviour, at least in the form proposed by Schapery. ${ }^{16}$ Such an extension is the subject of this paper.

\section{NON-LINEAR VISCOELASTIC MODEL}

\section{Constitutive relations}

The focus of this paper is the development of an efficient time integration procedure, within an implicit finite element analysis context, for small-strain, anisotropic, non-linear viscoelasticity. In order to motivate the constitutive functional form adopted in this work, the 1-D non-linear viscoelastic characterization proposed by Schapery ${ }^{16}$ is first reviewed.

The defining characteristic of linear viscoelasticity is that, when a constant strain is imposed at $t=0$ (relaxation test), the ratio of stress response to strain input is a function only of time, i.e.

$$
\sigma=E^{\infty} \varepsilon+\Delta E(t) \varepsilon
$$

Here $E^{\infty}$ is the equilibrium modulus and $\Delta E(t)$, which vanishes as $t \rightarrow \infty$, is independent of the strain magnitude. For non-constant strain history, a superposition principle leads to the familiar hereditary integral representation:

$$
\sigma=E^{\infty} \varepsilon+\int_{0}^{t} \Delta E(t-\tau) \frac{\mathrm{d} \varepsilon}{\mathrm{d} \tau} \mathrm{d} \tau
$$


Generalizing to the non-linear regime, Schapery ${ }^{16}$ proposed the following functional form:

$$
\sigma=h_{e} E^{\infty} \varepsilon+h_{1} \int_{0}^{t} \Delta E\left(\rho-\rho^{\prime}\right) \frac{\mathrm{d} h_{2} \varepsilon}{\mathrm{d} \tau} \mathrm{d} \tau
$$

where $h_{e}, h_{1}, h_{2}$ are functions of strain, ${ }^{\S}$ and $\rho$ is some strain-driven 'reduced time', defined by

$$
\rho=\int_{0}^{t} \frac{\mathrm{d} t^{\prime}}{A_{\varepsilon}\left[\varepsilon\left(t^{\prime}\right)\right]}
$$

The definition for $\rho^{\prime}$ is analogous with $\tau$ being the upper limit of integration. Variations in $h_{e}, h_{1}$, $h_{2}$ are due to third- and higher-order strain effects in the Helmholtz free energy, while changes in $A_{\varepsilon}$ arise from similar strong strain influences in both entropy production and free energy.

Turning now to 3-D anisotropic viscoelasticity, the linear formulation, adopted in Reference 15 , can be cast in the form

$$
\begin{aligned}
\sigma_{i j}(t) & =\int_{0}^{t} \sum_{k} \sum_{l}\left[C_{i j k l}^{\infty}+\Delta C_{i j k l}\left(\xi_{i j k l}-\xi_{i j k l}^{\prime}\right)\right] \frac{\mathrm{d}}{\mathrm{d} t^{\prime}} \bar{\varepsilon}_{k l} \mathrm{~d} t^{\prime} \\
& =\left[\sum_{k} \sum_{l} C_{i j k l}^{\infty} \bar{\varepsilon}_{k l}\right]+\int_{0}^{t} \sum_{k} \sum_{l}\left[\Delta C_{i j k l}\left(\xi_{i j k l}-\xi_{i j k l}^{\prime}\right) \frac{\mathrm{d}}{\mathrm{d} t^{\prime}} \bar{\varepsilon}_{k l}\right] \mathrm{d} t^{\prime}
\end{aligned}
$$

where $C_{i j k l}^{\infty}$ are the equilibrium moduli, $\Delta C_{i j k l}$ the transient decay. $\bar{\varepsilon}_{k l}$ is the mechanical (total minus thermal) strain, i.e.

$$
\begin{aligned}
\bar{\varepsilon}_{k l} & =\varepsilon_{k l}-\hat{\varepsilon}_{k l} \\
& =\varepsilon_{k l}-\int_{T_{0}}^{T} b_{k l}(s) \mathrm{d} s
\end{aligned}
$$

with $b_{k l}$ being the anisotropic thermal expansion coefficients.

Thermal rheological simplicity is assumed in this work where the influence of temperature is encapsulated in a direction-dependent reduced time $\xi_{i j k l}$, defined in the usual manner as

$$
\xi_{i j k l}=\int_{0}^{t} \frac{\mathrm{d} t^{\prime}}{A_{i j k l}\left(T\left(t^{\prime}\right)\right)}
$$

Following the Schapery 1-D pattern shown in equation (3), non-linearity is introduced into the constitutive relation of equation (5), resulting in

$$
\sigma_{i j}(t)=h_{e}\left[\sum_{k} \sum_{l} C_{i j k l}^{\infty} \bar{\varepsilon}_{k l}\right]+h_{1} \int_{0}^{t} \sum_{k} \sum_{l}\left[\Delta C_{i j k l}\left(\xi_{i j k l}-\xi_{i j k l}^{\prime}\right) \frac{\mathrm{d}}{\mathrm{d} t^{\prime}}\left(h_{2} \bar{\varepsilon}_{k l}\right)\right] \mathrm{d} t^{\prime}
$$

where the scalar functions $h_{e}, h_{1}, h_{2}$ are in general dependent on the entire mechanical strain tensor. The reduced time is also influenced by the mechanical strain via

$$
\xi_{i j k l}=\int_{0}^{t} \frac{\mathrm{d} t}{A_{i j k l}\left(T\left(t^{\prime}\right), \overline{\mathbf{E}}\left(t^{\prime}\right)\right)}
$$

\footnotetext{
${ }^{\S}$ The use of strain as the independent variable conforms to the present relaxation-based formulation. Alternative creep compliance-based formulations would have used the stress as the independent variable for the non-linear parameters $h_{e}$, $h_{1}, h_{2}$
} 
Viscoelastic stress relaxation behaviour is typically described by a Prony exponential series as

$$
C_{i j k l}\left(\xi_{i j k l}\right)=C_{i j k l}^{\infty}+\sum_{m=1}^{N} \tilde{C}_{i j k l m} \exp \left(-\frac{\xi_{i j k l}}{\tau_{i j k l m}}\right)
$$

In the following only one exponential term is adopted. This simplifies the algebra and focuses attention on the main ideas. Generalization to multiple exponential terms is straightforward and illustrated later. Substitution of the one exponential term decay yields

$$
\Delta C_{i j k l}\left(\xi_{i j k l}\right)=\tilde{C}_{i j k l} \exp \left(-\frac{\xi_{i j k l}}{\tau_{i j k l}}\right)
$$

Substituting equation (10) into (7)

$$
\sigma_{i j}(t)=h_{e}\left[\sum_{k} \sum_{l} C_{i j k l}^{\infty} \bar{\varepsilon}_{k l}\right]+h_{1} \int_{0}^{t} \sum_{k} \sum_{l}\left[\tilde{C}_{i j k l} \exp \left(-\frac{\xi_{i j k l}-\xi_{i j k l}^{\prime}}{\tau_{i j k l}}\right) \frac{\mathrm{d}}{\mathrm{d} t^{\prime}}\left(h_{2} \bar{\varepsilon}_{k l}\right)\right] \mathrm{d} t^{\prime}
$$

Choice of state variables

The proper choice of state variables is an important aspect in the design of the present viscoelastic-time-integration procedure. Ideally, the evolution laws for those variables should be well defined, and be amenable to time integration by stable algorithms such as backward Euler. First, the constitutive relation of equation (11) is rewritten as follows:

$$
\sigma_{i j}(t)=h_{e}\left[\sum_{k} \sum_{l} C_{i j k l}^{\infty} \bar{\varepsilon}_{k l}\right]+h_{1} \sum_{k} \sum_{l} \int_{0}^{t} \tilde{C}_{i j k l}\left[1-\left(1-\exp \left(-\frac{\xi_{i j k l}-\xi_{i j k l}^{\prime}}{\tau_{i j k l}}\right)\right)\right] \frac{\mathrm{d}}{\mathrm{d} t^{\prime}}\left(h_{2} \bar{\varepsilon}_{k l}\right) \mathrm{d} t^{\prime}
$$

Although not obvious at first sight, $D_{i j k l}$, as defined below, was found to fulfil the above criteria for choosing state variables.

$$
D_{i j k l}=\int_{0}^{t} \tilde{C}_{i j k l}\left[1-\exp \left(-\frac{\xi_{i j k l}-\xi_{i j k l}^{\prime}}{\tau_{i j k l}}\right)\right] \frac{\mathrm{d}}{\mathrm{d} t^{\prime}}\left(h_{2} \bar{\varepsilon}_{k l}\right) \mathrm{d} t^{\prime}
$$

Note that $D_{i j k l}$ exhibits minor symmetry, i.e. $D_{i j k l}=D_{j i k l}=D_{i j l k}$, so that there are at most 36 distinct scalars to track in the general anisotropic case. With this definition, the stress can be expressed in a form without integral signs

$$
\sigma_{i j}(t)=h_{e}\left[\sum_{k} \sum_{l} C_{i j k l}^{\infty} \bar{\varepsilon}_{k l}\right]+h_{1} \sum_{k} \sum_{l}\left[\tilde{C}_{i j k l} h_{2} \bar{\varepsilon}_{k l}-D_{i j k l}\right]
$$

The immediate task is to derive an evolution law for $D_{i j k l}$. To this end, a special differentiation formula is needed.

\section{A differentiation formula}

In preparation for deriving the evolution law of $D_{i j k l}$, a formula for differentiating functions of the form

$$
y(\tau)=\int_{0}^{t} f\left(\tau, \tau^{\prime}\right) \mathrm{d} \tau^{\prime}
$$

with respect to $\tau$, was developed. 
Denoting by $\Delta \tau$ an infinitesimal change in $\tau$, the following is observed:

$$
\begin{aligned}
y(\tau & +\Delta \tau)-y(\tau) \\
& =\int_{0}^{\tau+\Delta \tau} f\left(\tau+\Delta \tau, \tau^{\prime}\right) \mathrm{d} \tau^{\prime}-\int_{0}^{\tau} f\left(\tau, \tau^{\prime}\right) \mathrm{d} \tau^{\prime} \\
& =\int_{\tau}^{\tau+\Delta \tau} f\left(\tau+\Delta \tau, \tau^{\prime}\right) \mathrm{d} \tau^{\prime}+\int_{0}^{\tau}\left[f\left(\tau+\Delta \tau, \tau^{\prime}\right)-f\left(\tau, \tau^{\prime}\right)\right] \mathrm{d} \tau^{\prime} \\
& \approx \int_{\tau}^{\tau+\Delta \tau}\left(f\left(\tau, \tau^{\prime}\right)+\frac{\partial f}{\partial x}\left(\tau, \tau^{\prime}\right) \Delta \tau\right) \mathrm{d} \tau^{\prime}+\int_{0}^{\tau} \frac{\partial f}{\partial x}\left(\tau, \tau^{\prime}\right) \Delta \tau \mathrm{d} \tau^{\prime} \\
& \approx f(\tau, \tau) \Delta \tau+\frac{\partial f}{\partial x}(\tau, \tau)(\Delta \tau)^{2}+\Delta \tau \int_{0}^{\tau} \frac{\partial f}{\partial x}\left(\tau, \tau^{\prime}\right) \mathrm{d} \tau^{\prime}
\end{aligned}
$$

where $\partial f / \partial x$ denotes the partial derivative of $f$ w.r.t. its first argument. Following the definition of the derivative,

$$
\begin{aligned}
\frac{\mathrm{d} y}{\mathrm{~d} \tau} & =\lim _{\Delta \tau \rightarrow 0} \frac{y(\tau+\Delta \tau)-y(\tau)}{\Delta \tau} \\
& =f(\tau, \tau)+\int_{0}^{\tau} f(\tau, \tau)+\int_{0}^{\tau} \frac{\partial f}{\partial x}\left(\tau, \tau^{\prime}\right) \mathrm{d} \tau^{\prime}
\end{aligned}
$$

\section{Time evolution law for $D_{i j k l}$}

Examining the definition of $D_{i j k l}$ in equation (13), one notices that $\xi_{i j k l}$ is a function of $t$ via the reduced time definition in equation (8). Similarly $\xi_{i j k l}^{\prime}$ is a function of $t^{\prime}$. So is the derivative (w.r.t. dummy time) at the end of the integrand. The functional form $f\left(t, t^{\prime}\right)$ is observed for the integrand and the differentiation formula just developed can be applied. In this particular case,

$$
\begin{aligned}
f(t, t) & =0 \\
\frac{\partial f}{\partial x} & =\tilde{C}_{i j k l} \exp \left(-\frac{\xi_{i j k l}-\xi_{i j k l}^{\prime}}{\tau_{i j k l}}\right) \frac{1}{\tau_{i j k l}} \frac{1}{A_{i j k l}} \frac{\mathrm{d}}{\mathrm{d} t^{\prime}}\left(h_{2} \bar{\varepsilon}_{k l}\right)
\end{aligned}
$$

where the $1 / A_{i j k l}$ factor comes from differentiation of equation (8) and $A_{i j k l}$ is to be evaluated at time $t$. Finally, the time evolution law takes the form

$$
\begin{aligned}
\frac{\mathrm{d}}{\mathrm{d} t} D_{i j k l} & =\frac{1}{\tau_{i j k l} A_{i j k l}} \int_{0}^{t} \tilde{C}_{i j k l} \exp \left(-\frac{\xi_{i j k l}-\xi_{i j k l}^{\prime}}{\tau_{i j k l}}\right) \frac{\mathrm{d}}{\mathrm{d} t^{\prime}}\left(h_{2} \bar{\varepsilon}_{k l}\right) \mathrm{d} t^{\prime} \\
& =\frac{1}{\tau_{i j k l} A_{i j k l}} \int_{0}^{t} \tilde{C}_{i j k l}\left[1-\left(1-\exp \left(-\frac{\xi_{i j k l}-\xi_{i j k l}^{\prime}}{\tau_{i j k l}}\right) \frac{\mathrm{d}}{\mathrm{d} t^{\prime}}\left(h_{2} \bar{\varepsilon}_{k l}\right) \mathrm{d} t^{\prime}\right.\right. \\
& =\frac{1}{\tau_{i j k l} A_{i j k l}}\left[\tilde{C}_{i j k l} h_{2} \bar{\varepsilon}_{k l}-D_{i j k l}\right]
\end{aligned}
$$




\section{Summary}

The present constitutive law is summarized as follows:

where

$$
\sigma_{i j}(t)=h_{e}\left[\sum_{k} \sum_{l} C_{i j k l}^{\infty} \bar{\varepsilon}_{k l}\right]+h_{1} \sum_{k} \sum_{l}\left[\tilde{C}_{i j k l} h_{2} \bar{\varepsilon}_{k l}-D_{i j k l}\right]
$$

$$
D_{i j k l}=\int_{0}^{t} \tilde{C}_{i j k l}\left[1-\exp \left(-\frac{\xi_{i j k l}-\xi_{i j k l}^{\prime}}{\tau_{i j k l}}\right)\right] \frac{\mathrm{d}}{\mathrm{d} t^{\prime}}\left(h_{2} \bar{\varepsilon}_{k l}\right) \mathrm{d} t^{\prime}
$$

with its time evolution

$$
\frac{\mathrm{d}}{\mathrm{d} t} D_{i j k l}=\frac{1}{\tau_{i j k l} A_{i j k l}}\left[\tilde{C}_{i j k l} h_{2} \bar{\varepsilon}_{k l}-D_{i j k l}\right]
$$

The achievement of such differential (rate) form for the evolution of state variables is the high point in the present algorithmic development. It offers the user great flexibility in choosing a suitable time integration method, such as backward-Euler or trapozoidal rule, based on ease of coding and accuracy considerations. Perhaps more importantly, it obviates the need for nonphysical assumptions such as linear time variation of strain throughout the increment. Such assumptions are adopted in some viscoelastic constitutive updating schemes such as the one in Reference 7.

It should be remarked that the algorithmic development in this work bears a striking similarity to the linear viscoelastic case presented in Reference 15. The relative ease with which the original approach can be generalized to the Schapery non-linear situation is a testament to the versatility of the method.

\section{Generalization to multiple exponential terms} form

It suffices to illustrate the case for two exponential terms, where the transient decay takes the

$$
\Delta C_{i j k l}\left(\xi_{i j k l}\right)=\tilde{C}_{i j k l} \exp \left(-\frac{\xi_{i j k l}}{\tilde{\tau}_{i j k l}}\right)+\hat{C}_{i j k l} \exp \left(-\frac{\xi_{i j k l}}{\hat{\tau}_{i j k l}}\right)
$$

The net effect of this modification is the addition of one more term in equation (12), resulting in the following:

$$
\begin{aligned}
\sigma_{i j}(t)= & h_{e}\left[\sum_{k} \sum_{l} C_{i j k l}^{\infty} \bar{\varepsilon}_{k l}\right]+h_{1} \sum_{k} \sum_{l} \int_{0}^{t} \tilde{C}_{i j k l}\left[1-\left(1-\exp \left(-\frac{\xi_{i j k l}-\xi_{i j k l}^{\prime}}{\tilde{\tau}_{i j k l}}\right)\right)\right] \frac{\mathrm{d}}{\mathrm{d} t^{\prime}}\left(h_{2} \bar{\varepsilon}_{k l}\right) \mathrm{d} t^{\prime} \\
& +h_{1} \sum_{k} \sum_{l} \int_{0}^{t} \hat{C}_{i j k l}\left[1-\left(1-\exp \left(-\frac{\xi_{i j k l}-\xi_{i j k l}^{\prime}}{\hat{\tau}_{i j k l}}\right)\right)\right] \frac{\mathrm{d}}{\mathrm{d} t^{\prime}}\left(h_{2} \bar{\varepsilon}_{k l}\right) \mathrm{d} t^{\prime}
\end{aligned}
$$

Naturally, one defines the following state variables:

$$
\begin{aligned}
D_{i j k l} & =\int_{0}^{t} \tilde{C}_{i j k l}\left[1-\exp \left(-\frac{\xi_{i j k l}-\xi_{i j k l}^{\prime}}{\tilde{\tau}_{i j k l}}\right)\right] \frac{\mathrm{d}}{\mathrm{d} t^{\prime}}\left(h_{2} \bar{\varepsilon}_{k l}\right) \mathrm{d} t^{\prime} \\
E_{i j k l} & =\int_{0}^{t} \hat{C}_{i j k l}\left[1-\exp \left(-\frac{\xi_{i j k l}-\xi_{i j k l}^{\prime}}{\hat{\tau}_{i j k l}}\right)\right] \frac{\mathrm{d}}{\mathrm{d} t^{\prime}}\left(h_{2} \bar{\varepsilon}_{k l}\right) \mathrm{d} t^{\prime}
\end{aligned}
$$


so that the stress can be expressed in a form without integral signs as

$$
\sigma_{i j}(t)=h_{e}\left[\sum_{k} \sum_{l} C_{i j k l}^{\infty} \bar{\varepsilon}_{k l}\right]+h_{1} \sum_{k} \sum_{l}\left[\left(\tilde{C}_{i j k l}+\hat{C}_{i j k l}\right) h_{2} \bar{\varepsilon}_{k l}-D_{i j k l}-E_{i j k l}\right]
$$

Clearly, the evolution laws for $D_{i j k l}$ and $E_{i j k l}$ are completely analogous to that described before in the time evolution law section.

To summarize, the addition of more exponential terms in the Prony series translates into more storage requirements and the added book keeping costs needed to update the extra state variables, but introduces no conceptual difficulty.

\section{CONSTITUTIVE UPDATES IN A FINITE ELEMENT FRAMEWORK}

In a typical 'implicit' finite element program that employs non-linear material models, the discretized principle of virtual work, which enforces equilibrium and boundary conditions in a weak sense, generates an estimated incremental displacement field. Such displacement field is used to calculate integration point values of the stress and other field variables at the end of a time increment. If these stresses do not satisfy the principle of virtual work, then the estimate of the incremental displacement field is revised and new increment end stresses are calculated; iteration continues until the principle of virtual work is satisfied to within acceptable tolerances.

Within this context, the FEM program's 'constitutive update subroutine', being called once for each integration point for each global iteration, must perform the following functions:

Input:

1. The (converged values of) stress, strain, state variables, etc., at time $n$

2. The (estimated) strain and perhaps other field variables at time $n+1$

Output:

1. The stress and state variables at time $n+1$

2. The consistent tangent operator to be used in a Newton-type iterative method

\section{IMPLICIT TIME INTEGRATION}

Focusing on the computational task at hand and adding superscripts to indicate the time step, the present constitutive law can be re-written as

$$
\sigma_{i j}^{n+1}=h_{e}^{n+1}\left[\sum_{k} \sum_{l} C_{i j k l}^{\infty} \bar{\varepsilon}_{k l}^{n+1}\right]+h_{1}^{n+1} \sum_{k} \sum_{l}\left[\widetilde{C}_{i j k l} h_{2}^{n+1} \bar{\varepsilon}_{k l}^{n+1}-D_{i j k l}^{n+1}\right]
$$

Because of the 'Input' items discussed in the last section, the only unknown on the right-hand side is $D_{i j k l}^{n+1}$.

\footnotetext{
'E.g. temperature, if heat transfer is involved 
As mentioned before, the user is free to choose an appropriate method for integrating equation (18). The backward Euler scheme is implemented here for ease of coding. Alternate strategies such as the generalized trapezoidal rule ('alpha' $=0 \cdot 5$ ), can be adopted without difficulty.

Replacing the derivative by finite difference in equation (18), and using the backward Euler scheme, which is known for its stability, the following can be obtained:

$$
\frac{D_{i j k l}^{n+1}-D_{i j k l}^{n}}{\Delta t}=\frac{1}{\tau_{i j k l} A_{i j k l}}\left[\tilde{C}_{i j k l} h_{2}^{n+1} \bar{\varepsilon}_{k l}^{n+1}-D_{i j k l}^{n+1}\right]
$$

Rearranging yields

$$
D_{i j k l}^{n+1}=\left(\frac{1}{\Delta t}+\frac{1}{\tau_{i j k l} A_{i j k l}^{n+1}}\right)^{-1}\left[\frac{D_{i j k l}^{n}}{\Delta t}+\frac{\tilde{C}_{i j k l} h_{2}^{n+1} \bar{\varepsilon}_{k l}^{n+1}}{\tau_{i j k l} A_{i j k l}^{n+1}}\right]
$$

Note that this is almost identical in form to the linear case presented in Reference 15.

\section{CONSISTENT TANGENT OPERATOR}

The Consistent Tangent Operator (CTO) concept was introduced by Simo and Taylor ${ }^{17}$ in the context of rate-independent plasticity. During the incremental solution process for non-linear problems, non-linear virtual work equations are solved by Newton's method. In updating the global 'Jacobian' matrix, estimates are needed, at the integration point level, of the rate of change of stress w.r.t. strain.

Previously, the quantities $\partial \dot{\sigma} / \partial \dot{\varepsilon}$ were used, resulting in sub-optimal convergence rates. It turns out that using the quantity $\partial \sigma^{n+1} / \partial \Delta \varepsilon$ produces better results because this expression depends not only on the constitutive law but also on the time integration algorithm in the constitutive update. The use of this quantity, namely the CTO, preserves the quadratic convergence rate of Newton's method and accelerates the search for displacement fields that best satisfies the principle of virtual work.

Derivation of the CTO involves nothing more than a careful 'differentiation' of the constitutive update procedure. While conceptually simple, the present derivation is slightly more algebraically involved than the linear case described in Reference 15 . Since the intermediate details are purely algebraic in nature (mostly application of the product rule of differentiation), only the final result is shown below:

$$
\begin{aligned}
\frac{\partial \sigma_{i j}^{n+1}}{\partial \Delta \varepsilon_{a b}}= & h_{e} C_{i j a b}^{\infty}+\frac{\partial h_{e}}{\partial \Delta \varepsilon_{a b}}\left[\sum_{k} \sum_{l} C_{i j k l}^{\infty} \bar{\varepsilon}_{k l}^{n+1}\right]+\frac{\partial h_{1}}{\partial \Delta \varepsilon_{a b}} \sum_{k} \sum_{l}\left[\tilde{C}_{i j k l} h_{2} \bar{\varepsilon}_{k l}^{n+1}-D_{i j k l}^{n+1}\right] \\
& +h_{1} h_{2} \tilde{C}_{i j a b}-h_{1} h_{2} G_{i j a b}+h_{1} \frac{\partial h_{2}}{\partial \Delta \varepsilon_{a b}}\left[\sum_{k} \sum_{l} C_{i j k l} \bar{\varepsilon}_{k l}^{n+1}\right]-h_{1} \frac{\partial h_{2}}{\partial \Delta \varepsilon_{a b}}\left[\sum_{k} \sum_{l} G_{i j k l} \bar{\varepsilon}_{k l}^{n+1}\right]
\end{aligned}
$$

where

$$
G_{i j k l} \equiv\left(\frac{1}{\Delta t}+\frac{1}{\tau_{i j k l} A_{i j k l}^{n+1}}\right)^{-1} \frac{\tilde{C}_{i j k l}}{\tau_{i j k l} A_{i j k l}^{n+1}}
$$

In the above, quantities such as $h_{e}, h_{1}, h_{2}$, as well as their partial derivatives, are to be evaluated at the end of the increment. 
In the case of coupled temperature displacement analysis, the Consistent tangent operator w.r.t. temperature is also needed. The following expression is provided for completeness:

$$
\frac{\partial \sigma_{i j}^{n+1}}{\partial \Delta T}=-h_{e}\left[\sum_{k} \sum_{l} C_{i j k l}^{\infty} b_{k l}\right]-h_{1} h_{2}\left[\sum_{k} \sum_{l} \tilde{C}_{i j k l} b_{k l}\right]+h_{1} h_{2}\left[\sum_{k} \sum_{l} G_{i j k l} b_{k l}\right]
$$

\section{NUMERICAL EXAMPLES}

To illustrate how the current algorithm can be implemented in a general purpose FEM code, a 'user material' subroutine UMAT, which serves precisely the function of the constitutive update discussed before, was developed. The FEM code ABAQUS was chosen and linked with this subroutine.

Examples 1 and 2 serve to validate the anisotropic, non-linear capability of the user subroutine, using very simple loading conditions of triaxial tension and simple shear. Example 3 involves a more general loading condition with stress gradients, namely a cantilever beam loaded at the tip.

For Examples 1 and 2, the hypothetical viscoelastic material was chosen to be orthotropic, with principal directions along the 1,2 and 3 axes. The glassy and equilibrium engineering constants are shown in Table I. Material characterizations for Example 3 will be described later.

Note that $v_{i j}$ is the Poisson's ratio that characterizes the transverse strain in the $j$-direction, when the material is stressed in the $i$-direction. In general, $v_{i j}$ is not equal to $v_{j i}$ they are related by $v_{i j} / E_{i}=v_{j i} / E_{j}$.

These values completely determine the equilibrium relaxation moduli $C_{i j k l}^{\infty}$ and the glassy relaxation moduli $C_{i j k l}^{0}=C_{i j k l}^{\infty}+\tilde{C}_{i j k l}$. The values for $\tilde{C}_{i j k l}$ can be obtained easily by subtraction.

The time period of simulation was chosen as $0 \leqslant t \leqslant 1$. Also, the relaxation times were set to

$$
\begin{aligned}
\tau_{2222} & =0.09 \\
\tau_{3333} & =0.08 \\
\tau_{1313} & =\tau_{1331}=\tau_{3113}=\tau_{3131}=0.09 \\
\tau_{2323} & =\tau_{2332}=\tau_{3223}=\tau_{3232}=0.08
\end{aligned}
$$

Table I. Glassy and equilibrium moduli

\begin{tabular}{lcc}
\hline Moduli & Glassy value & $\begin{array}{c}\text { Equilibrium } \\
\text { value }\end{array}$ \\
\hline$E_{1}$ & $2 \cdot 8$ & $0 \cdot 23$ \\
$E_{2}$ & $2 \cdot 7$ & $0 \cdot 22$ \\
$E_{3}$ & $2 \cdot 6$ & $0 \cdot 21$ \\
$v_{21}$ & $0 \cdot 3$ & $0 \cdot 3$ \\
$v_{31}$ & $0 \cdot 3$ & $0 \cdot 3$ \\
$v_{32}$ & $0 \cdot 3$ & $0 \cdot 3$ \\
$G_{12}$ & 1 & $0 \cdot 08$ \\
$G_{13}$ & $0 \cdot 9$ & $0 \cdot 075$ \\
$G_{23}$ & $0 \cdot 8$ & $0 \cdot 07$ \\
\hline
\end{tabular}


and

$$
\tau_{i j k l}=0 \cdot 1 \quad \text { for the rest }
$$

In the interest of obtaining manageable analytical solutions, the effects of temperature change, which causes reduced time shifting and thermal strains, were not considered. The anisotropic model takes the simpler form

$$
\sigma_{i j}(t)=h_{e}\left[\sum_{k} \sum_{l} C_{i j k l}^{\infty} \varepsilon_{k l}\right]+h_{1} \int_{0}^{t} \sum_{k} \sum_{l}\left[\tilde{C}_{i j k l} \exp \left(-\frac{t-t^{\prime}}{\tau_{i j k l}}\right) \frac{\mathrm{d}}{\mathrm{d} t^{\prime}}\left(h_{2} \varepsilon_{k l}\right)\right] \mathrm{d} t^{\prime}
$$

where $h_{e}, h_{1}, h_{2}$ are functions of all six strain components $\varepsilon_{11}, \ldots, \varepsilon_{23}$. There is a requirement that at zero strain, $h_{e}=h_{1}=h_{2}=1$, so that the model coincides with linear viscoelasticity. In this work, the following simple functional forms are arbitrarily assigned:

$$
\begin{aligned}
& h_{e}=1-\alpha\left(\varepsilon_{11}+\varepsilon_{22}+\varepsilon_{33}+\varepsilon_{12}+\varepsilon_{13}+\varepsilon_{23}\right) \\
& h_{1}=1-\beta\left(\varepsilon_{11}+\varepsilon_{22}+\varepsilon_{33}+\varepsilon_{12}+\varepsilon_{13}+\varepsilon_{23}\right) \\
& h_{2}=1-\gamma\left(\varepsilon_{11}+\varepsilon_{22}+\varepsilon_{33}+\varepsilon_{12}+\varepsilon_{13}+\varepsilon_{23}\right)
\end{aligned}
$$

where $\alpha=200, \beta=100, \gamma=50$.

Examples 1 and 2 are verification problems where numerical results produced by incorporating the present algorithm in a general purpose FEM code are compared against analytical solutions. Before proceeding, it is important to point out that the non-linear nature of the constitutive relation prevents the 'inversion' of relaxation moduli to get creep compliances. In other words, given the relaxation-based expression

$$
\sigma=h_{e} E^{\infty} \varepsilon+h_{1} \int_{0}^{t} \Delta E\left(\rho-\rho^{\prime}\right) \frac{\mathrm{d} h_{2} \varepsilon}{\mathrm{d} \tau} \mathrm{d} \tau
$$

it may not be possible to deduce the equivalent creep compliance-based expression

$$
\varepsilon=g_{0} D^{0} \sigma+g_{1} \int_{0}^{t} \Delta D\left(\psi-\psi^{\prime}\right) \frac{\mathrm{d} g_{2} \sigma}{\mathrm{d} \tau} \mathrm{d} \tau
$$

In fact, Schapery ${ }^{16}$ pointed out that such an expression may not exist - it is possible that the non-linear material response cannot be cast in both forms at the same time.

Because of this, and the fact that our constitutive formulation is relaxation-based, verification Examples 1 and 2 are both strain driven to make analytical solutions possible. The finite element implementation can, of course, handle traction driven problems. Such is the case for Example 3.

Example 1 (Imposed axial strain in one direction). Here, the material sample is subjected to an imposed strain $\varepsilon(t)$ in the one direction, and zero strain in other directions. The non-zero stress components are $\sigma_{11}, \sigma_{22}, \sigma_{33}$ (see Figures 1-3) (triaxial tension). The linear strain history is prescribed as

$$
\varepsilon(t)=\frac{t}{1000} \quad 0 \leqslant t \leqslant 1
$$




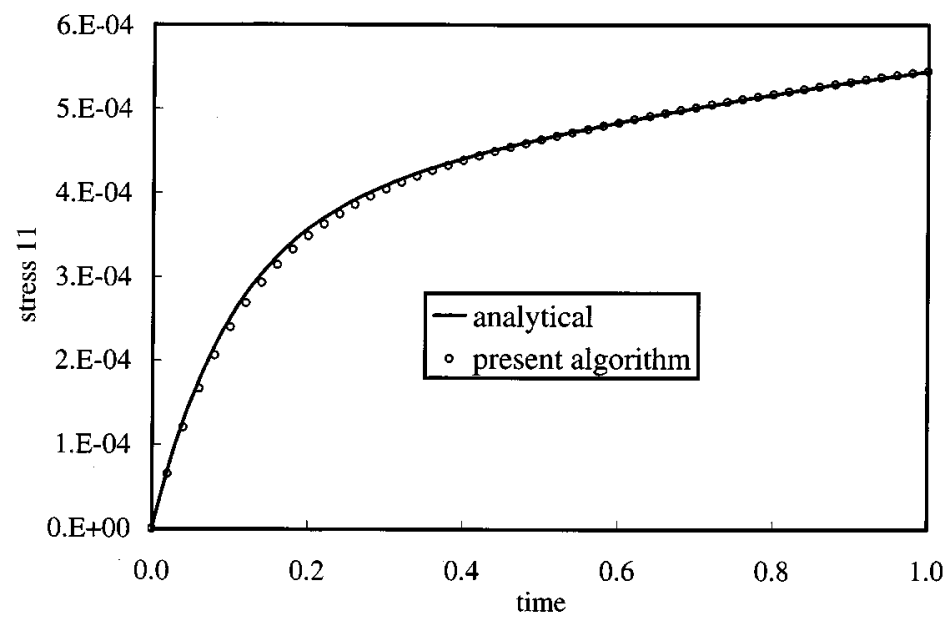

Figure 1. Example 1: Stress 11

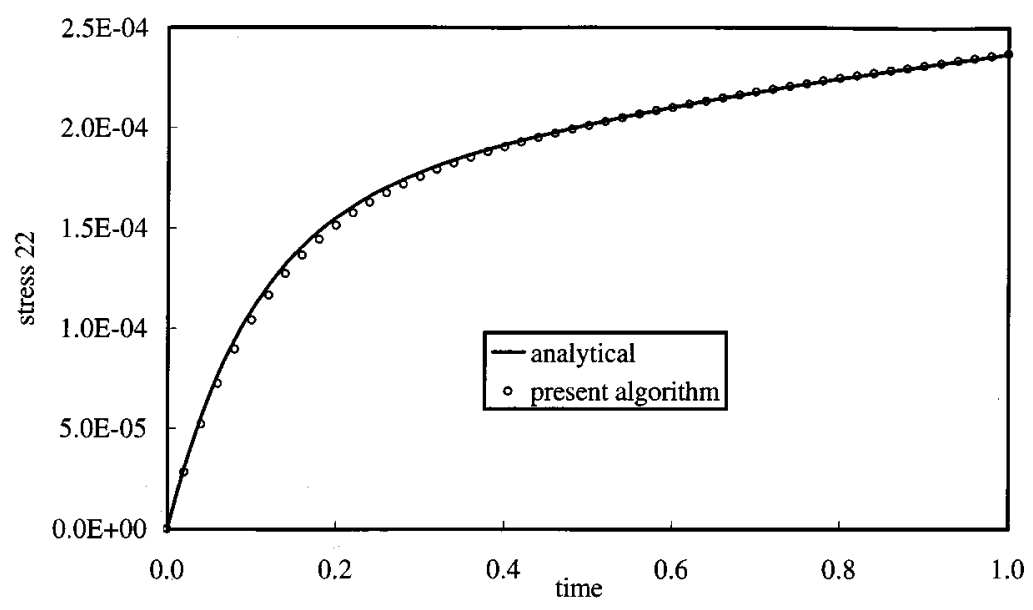

Figure 2. Example 1: Stress 22

The analytical solution becomes

$$
\begin{aligned}
& \sigma_{11}(t)=h_{e} C_{1111}^{\infty} \varepsilon+h_{1} \int_{0}^{t} \tilde{C}_{1111} \exp \left(-\frac{t-t^{\prime}}{\tau_{1111}}\right) \frac{\mathrm{d}}{\mathrm{d} t^{\prime}}\left(h_{2} \varepsilon\right) \mathrm{d} t^{\prime} \\
& \sigma_{22}(t)=h_{e} C_{2211}^{\infty} \varepsilon+h_{1} \int_{0}^{t} \tilde{C}_{2211} \exp \left(-\frac{t-t^{\prime}}{\tau_{2211}}\right) \frac{\mathrm{d}}{\mathrm{d} t^{\prime}}\left(h_{2} \varepsilon\right) \mathrm{d} t^{\prime} \\
& \sigma_{33}(t)=h_{e} C_{3311}^{\infty} \varepsilon+h_{1} \int_{0}^{t} \tilde{C}_{3311} \exp \left(-\frac{t-t^{\prime}}{\tau_{3311}}\right) \frac{\mathrm{d}}{\mathrm{d} t^{\prime}}\left(h_{2} \varepsilon\right) \mathrm{d} t^{\prime}
\end{aligned}
$$




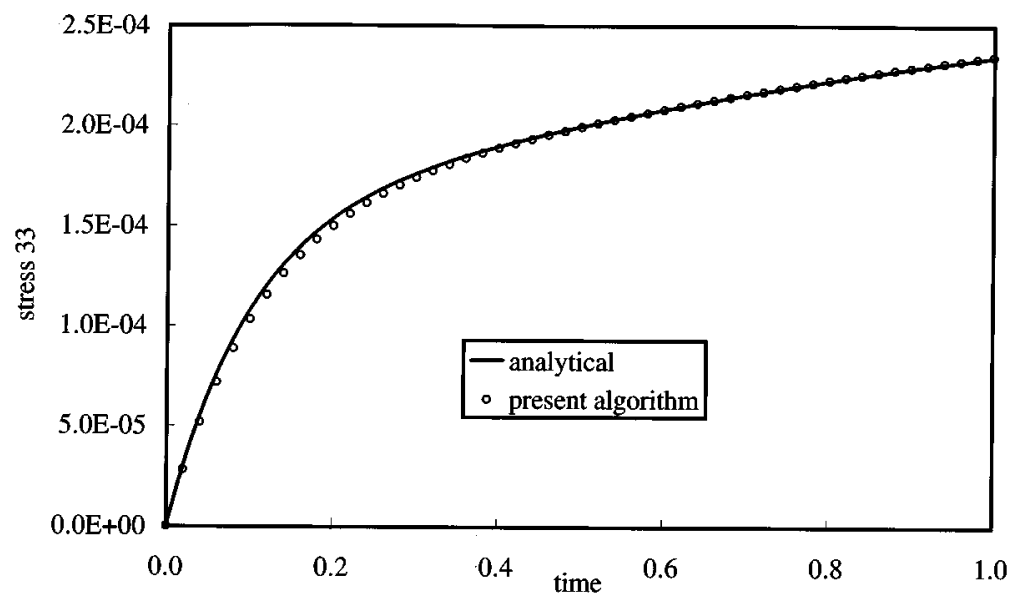

Figure 3. Example 1: Stress 33

which is readily computable given the simple functional forms for $h_{e}, h_{1}, h_{2}$ and $\varepsilon$. The software package Mathematica is used to perform such integrations.

The present time integration scheme was validated by performing a finite element analysis of a three-dimensional problem with 50 equal increments.

Example 2 (Simple shear). Here, the material sample is subjected to an applied shear strain

$$
\varepsilon_{12}(t)=\frac{t}{1000} \quad 0 \leqslant t \leqslant 1
$$

The analytical solution becomes (see Figure 4)

$$
\sigma_{12}(t)=2 h_{e} C_{1212}^{\infty} \varepsilon_{12}+2 h_{1} \int_{0}^{t} \tilde{C}_{1212} \exp \left(-\frac{t-t^{\prime}}{\tau_{1212}}\right) \frac{\mathrm{d}}{\mathrm{d} t^{\prime}}\left(h_{2} \varepsilon_{12}\right) \mathrm{d} t^{\prime}
$$

Again, a 3-D FEM analysis incorporating the present time integration procedure was performed with 50 equal increments.

Example 3 (Beam with tip load). This example involves a more complex problem with spatially varying stress states. A cantilever beam is subjected to a transient concentrated tip load

$$
P=P_{0}\left[H(t)-H\left(t-t_{1}\right)\right]
$$

where $P_{0}=1 \mathrm{~N}, t_{1}=10 \mathrm{~s}$, and $H$ is the Heavyside function. The beam has a length, $L$, of $18 \mathrm{~m}$ and a square cross-section of area $A=1 \mathrm{~m}^{2}$.

Had this been a purely elastic analysis, the classical Euler-Bernoulli beam theory would have predicted the following tip displacement (when loaded)

$$
w_{L}=\frac{P_{0} L^{3}}{3 E I}
$$

where $I$ is the area moment of inertia of the cross-section (in this case $1 / 12 \mathrm{~m}^{4}$ ). 


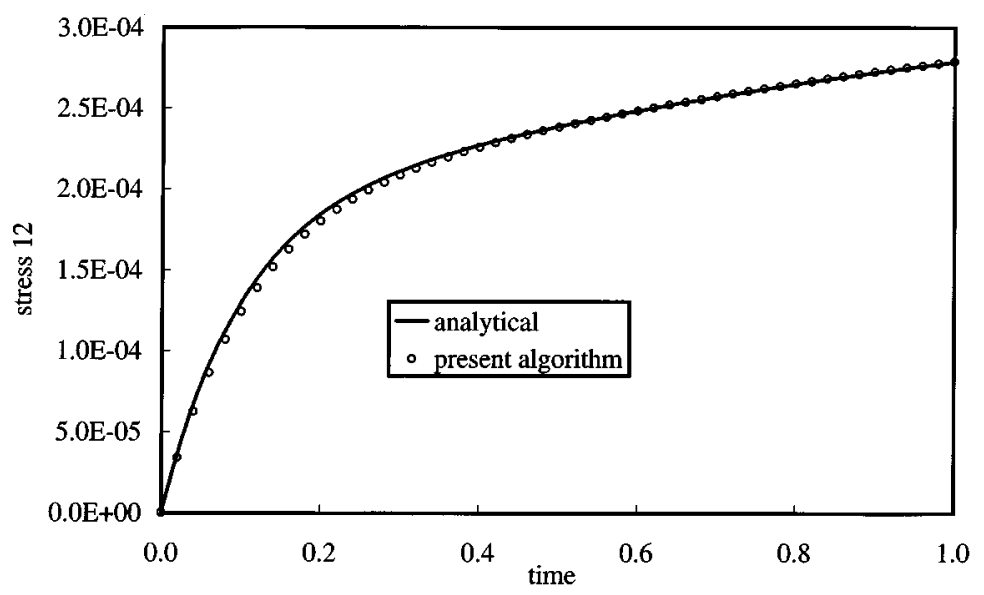

Figure 4. Example 2: Stress 12

Next, if linear viscoelasticity is assumed, i.e. $h_{e}=h_{1}=h_{2}=1$, the correspondence principle is often a powerful tool for predicting structural response. Suppose the hypothetical viscoelastic material has the following relaxation behaviour for the Young's modulus:

$$
E(t)=E_{\infty}+E_{1} \exp (-t / \rho)
$$

where the values of $E_{\infty}, E_{1}$ and $\rho$ are taken to be $0 \cdot 1,0 \cdot 4 \mathrm{MPa}$ and $1 \mathrm{~s}$, respectively. Then, the principle predicts the following tip displacement time history:

$$
w_{L}=\frac{P_{0} L^{3}}{3 I}\left[D(t)-D\left(t-t_{1}\right) H\left(t-t_{1}\right)\right]
$$

where the creep compliance $D(t)$ is given by

$$
D(t)=D_{0}+D_{1}(1-\exp (-t / \lambda))
$$

with

$$
E_{0} \equiv E_{\infty}+E_{1}, \quad D_{0} \equiv \frac{1}{E_{0}}, \quad D_{1} \equiv \frac{1}{E_{\infty}}-\frac{1}{E_{0}}, \quad \lambda \equiv \frac{E_{0} \rho}{E_{\infty}}
$$

(It should be noted that the beam theory solution approaches the 3-D elasticity solution for slender beams such as the present one whose aspect ratio is 18.) This linear viscoelastic solution is shown by the solid line in Figure 5.

As a first check on the correctness of the present time integration algorithm, a special version of the user material subroutine was prepared where $h_{e}=h_{1}=h_{2}=1$, and $C_{i j k l}$ isotropic, with Young's modulus described in equation (20), and a constant Poisson's ratio of $0 \cdot 3$." The finite element results, shown by the circles in Figure 5, agree well with the exact solution.

\footnotetext{
"By virtue of the 1-D nature of classical beam theory, only the Young's modulus in the axial direction enters into the picture. Transverse Young's moduli and Poisson ratios do not matter as far as beam theory is concerned 


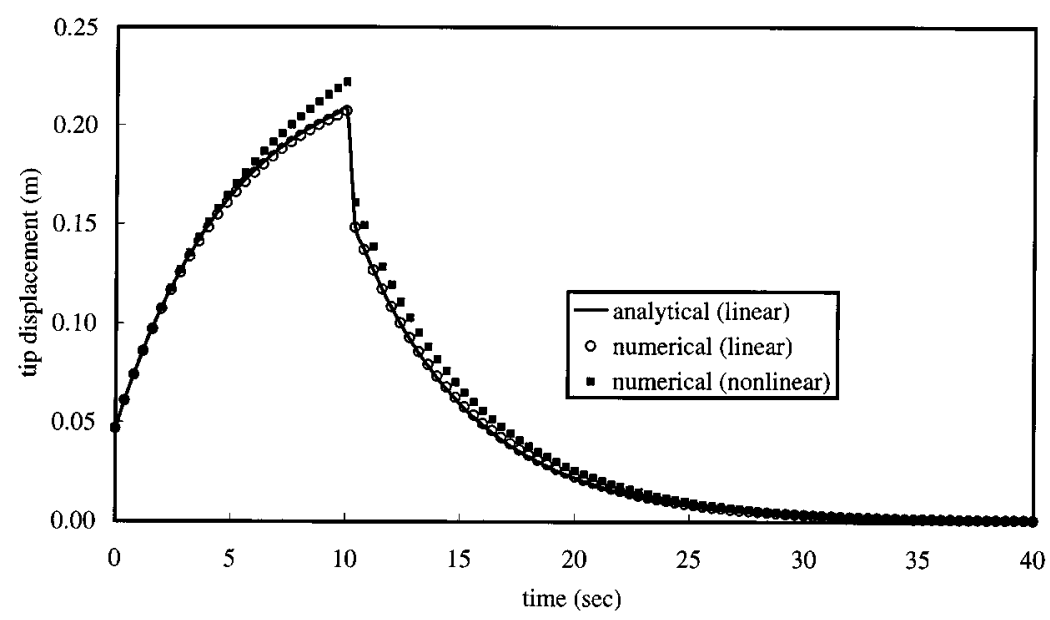

Figure 5. Example 3: Tip displacement

Finally, non-linear viscoelastic behaviour is explored. Non-linearity was introduced in the form of equation (19) with $\alpha=1200, \beta=50, \gamma=50$. The finite element results, shown by the squares, follow the same qualitative trend as the linear viscoelastic solution. Analytical solutions are not available in this non-linear case.

\section{CONCLUSION}

This study presents a natural extension to the non-linear viscoelastic regime, of a new time integration approach for anisotropic, thermal rheologically simple viscoelasticity, that was originally proposed by the authors. Among the key features of this algorithm are an intelligent choice of state variables leading to surprisingly simple evolution laws, and the ready incorporation into existing implicit, non-linear FEM codes. The generality of the method is illustrated in this work through the extension to Schapery's non-linear viscoelastic model. The algebraic modification is relatively minor, leaving intact the fundamental structure of the original scheme. Numerical examples involving both homogeneous and complicated stress states have been carried out, validating the present non-linear viscoelastic time integration procedure.

\section{REFERENCES}

1. R. L. Taylor, K. S. Pister and G. L. Goudreau, 'Thermomechanical analysis of viscoelastic solids', Int. J. Numer. Meth. Engng., 2, 45-59 (1970).

2. M. Henriksen, 'Non-linear viscoelastic stress analysis - a finite element approach', Comp. Struct., 18, 133-139 (1984).

3. J. C. Simo, 'On a fully three-dimensional finite strain viscoelastic damage model: formulation and computational aspects', Comp. Meth. Appl. Mech. Engng., 60, 153-173 (1987).

4. J. Lai and A. Bakker, '3-D schapery representation for non-linear viscoelasticity and finite element implementation', Comp. Mech., 18, 182-191 (1996).

5. M. Kaliske and H. Rothert, 'Formulation and implementation of three-dimensional viscoelasticity at small and finite strains', Comp. Mech., 19, 228-239 (1997). 
6. S. Yi, 'Finite element analysis of anisotropic viscoelastic composite structures and analytical determination of optimum viscoelastic material properties', Ph.D. Thesis, University of Illinois at Urbana - Champaign, 1991.

7. M. A. Zocher, S. E. Groves and D. H. Allen, 'A three-dimensional finite element formulation for thermoviscoelastic orthotropic media', Int. J. Numer. Meth. Engng., 40, 2267-2288 (1997).

8. S. Yi, G. Pollock, M. F. Ahmad and H. H. Hilton, 'Time dependent analysis of viscoelastic composite shell structures', Comp. Systems Engng., 3, 457-467 (1992).

9. S. Yi, K. H. Pierson and M. F. Ahmad, 'Parallel implementation of dynamic simulation to filamentary composite structures with general rate dependent damping', Comp. Systems Engng., 5, 469-477 (1994).

10. S. Yi, H. H. Hilton and M. F. Ahmad, 'Curing process induced viscoelastic residual stresses in polymer matrix laminated composites', ASME Int. Mech. Engng. Congr. Proc. ASME Mater. Div., (MD-Vol. 69-1), 65-76 (1995)).

11. S. Yi, H. H. Hilton and M. F. Ahmad, 'Non-linear thermo-viscoelastic analysis of interlaminar stresses in laminated composites', ASME J. Appl. Mech., 63(1), 218-224 (1996).

12. S. Yi, M. F. Ahmad and A. Ramesh, 'Data parallel computation for thermo-viscoelastic analysis of composite structures', Adv. Engng. Software, 27(N1-2), 97-102 (1996).

13. S. Yi, M. F. Ahmad and H. H. Hilton, 'Finite element algorithms for dynamic simulations of viscoelastic composite shell structures using conjugate gradient method on coarse grained and massively parallel machines', Int. J. Numer. Meth. Engng., 40, 1857-1875 (1997).

14. S. Yi, H. H. Hilton and M. F. Ahmad, 'Cure cycle simulations of composites with temperature and cure dependent anisotropic viscoelastic properties and stochastic delaminations', Mech. Composite Mater. Struct., 5(1), 81-101 (1998).

15. H. Poon and M. F. Ahmad, 'A material point time integration procedure for anisotropic, thermo rheologically simple, viscoelastic solids'. Comp. Mech., 21, 236-242 (1998).

16. R. A. Schapery, 'On the characterization of non-linear viscoelastic materials', Polym. Engng. Sci., 9, 295-310 (1969).

17. J. C. Simo and R. L. Taylor, 'Consistent tangent operators for rate-independent elastoplasticity', Comp. Meth. Appl. Mech. Engng., 48, 101-118 (1985). 